\title{
Equivalence between the Trefftz method and the method of fundamental solutions for Green's function of concentric spheres using the addition theorem and image concept
}

\author{
J. T. Chen ${ }^{1,2}$, H. C. Shieh ${ }^{1}$, J. J. Tsai ${ }^{1} \&$ J. W. Lee ${ }^{1}$ \\ ${ }^{I}$ Department of Harbor and River Engineering, \\ National Taiwan Ocean University, Taiwan \\ ${ }^{2}$ Department of Mechanical and Mechatronic Engineering, \\ National Taiwan Ocean University, Taiwan
}

\begin{abstract}
Following the success of the mathematical equivalence between the Trefftz method and the method of fundamental solutions for the annular Green's function, we extend to solve the Green's function of 3-D problems in this paper. The Green's function of the concentric sphere is first derived by using the image method which can be seen as a special case of method of fundamental solutions. Fixed-fixed boundary conditions are considered. Also, the Trefftz method is employed to derive the analytical solution by using the T-complete sets. By employing the addition theorem, both solutions are found to be mathematically equivalent when the number of Trefftz bases and the number of image points are both infinite. In the successive image process, the final two images freeze at the origin and infinity, where their singularity strengths can be analytically and numerically determined in a consistent manner. The agreement among the three results, including two analytical solutions by using the Trefftz method and the image method, and one numerical solution by using the conventional MFS is observed.
\end{abstract}

Keywords: Green's function, method of fundamental solutions, image method, Trefftz method. 


\section{Introduction}

In 1926, Trefftz presented the Trefftz method for solving boundary value problems by superimposing the functions which satisfy the governing equation [1]. The unknown coefficients are determined by matching the boundary condition. In the potential theory, it is well known that the method of fundamental solutions (MFS) can solve potential problems when a fundamental solution is known. This method was proposed by Kupradze [2] in Russia. The MFS can be viewed as an indirect boundary element method containing concentrated sources instead of boundary distributions. The initial idea is to approximate the solution through a linear combination of fundamental solutions where sources are located outside the domain of the problem. Moreover, it has certain advantages over BEM, e.g., no singularity and no boundary integrals are required. Bogomolny [3] studied the stability and error bound of the MFS. Li et al. [4] used the effective condition number to study the ill-poseness of collocation approaches, the MFS and the Trefftz method. They found that the condition number of the MFS is much worst than that of the Trefftz method. Although the Trefftz method and the MFS individually have a long history, the link between the two methods was not discussed in the literature until Chen et al.'s papers $[5,6]$. Researchers have paid attention to construct the mathematical relationship between the Trefftz method and MFS since 2006. For example, Schabck [7] found that the MFS with singularity at infinity behaves like the Trefftz base of harmonic polynomials. Chen et al. proved the equivalence between the Trefftz method and the MFS for Laplace and biharmonic problems containing a circular domain [5]. The key point is the use of the degenerate kernel or so-called the addition theorem. They only proved the equivalence by demonstrating a simple circle with angular distribution of singularity to link the two methods. Following the success of deriving the annular Green's function [6], we plan to derive the Green's function of a concentric sphere. Here, we also distribute singularities along the radial direction by using the method of image. Image solutions and Trefftz results for the annular Green's function were obtained [6]. Since a two-dimensional problem can be solved easily by using the complex variable, the image method can be seen as an alternative way to obtain the solution. However, the extension to 3-D problem is limited for the theory of complex variable. The image method can deal with the 3-D problems without any difficulty.

In this paper, we focus on proving the mathematical equivalence on the Green's functions for the Laplace problem of a concentric sphere derived by using the Trefftz method and the image method. Both surfaces of inner and outer boundaries are specified by the Dirichlet boundary conditions. By employing the image method and the addition theorem, the mathematical equivalence of the two solutions derived by using the Trefftz method and the image method will be proved when the number of successive image points and the number of the Trefftz bases are both infinite. The image method can be seen as a special case of the conventional MFS, since its image singularities locate outside the domain. The solution by using the image method for constructing the 2-D Green's 
function also indicates that a free constant is required for the completeness of the solution which is always neglected in the conventional MFS. In the 3-D case, the free constant becomes zero as the number of successive image points become infinity.

\section{Construction of the Green's function for the domain bounded by concentric spheres using the image method and the conventional MFS}

\subsection{The image solution}

For the problem of concentric sphere as shown in Fig. 1, the Green's function satisfies

$$
\nabla^{2} G(x, s)=\delta(x-s), x \in D,
$$

where $D$ is the domain of interest and $\delta$ denotes the Dirac-delta function for the source at $s$. For simplicity, the Green's function is considered to be subject to the Dirichlet boundary condition,

$$
G(x, s)=0, x \in B_{1} \cup B_{2},
$$

where $B_{1}$ and $B_{2}$ are the inner and outer boundaries of the sphere, respectively.
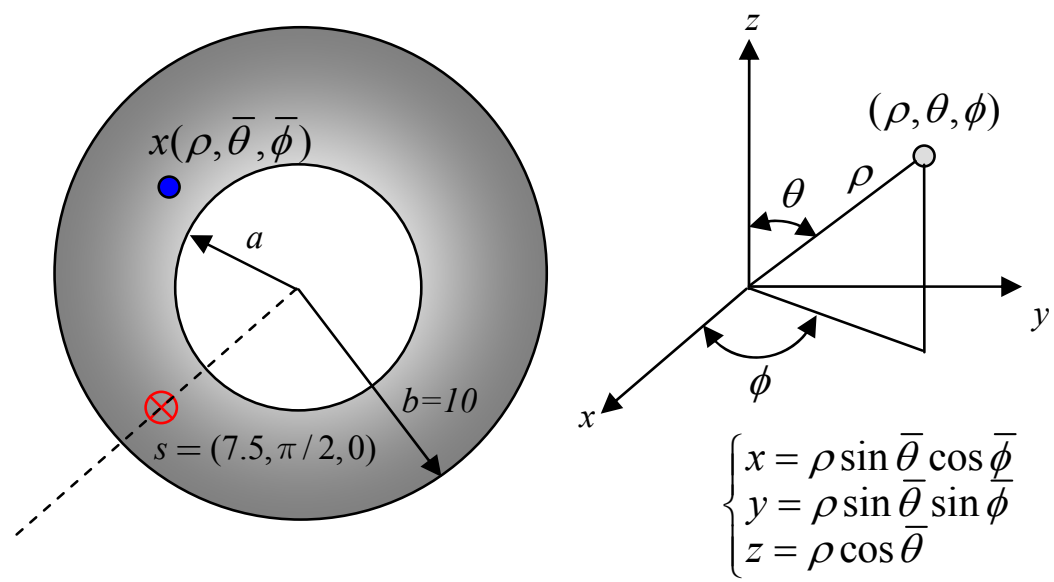

Figure 1: $\quad$ Sketch of an annular sphere subject to a concentrated load.

We consider the fundamental solution $U(s, x)$ for a source singularity which satisfies

$$
\nabla^{2} U(x, s)=\delta(x-s) .
$$

Then, we obtain the fundamental solution as follows:

$$
U(x, s)=\frac{-1}{4 \pi r},
$$


where $r$ is the distance between $s$ and $x(r \equiv|x-s|)$. Based on the separable property of the addition theorem or degenerate kernel, the fundamental solution $U(x, s)$ can be expanded into series form in the spherical coordinates:

$$
U(x, s)=\left\{\begin{array}{l}
\frac{-1}{4 \pi R_{s}} \\
-\sum_{n=1}^{\infty} \sum_{m=0}^{n} \frac{\boldsymbol{\varepsilon}_{m}}{4 \pi} \frac{(n-m) !}{(n+m) !} \cos (m(\overline{\boldsymbol{\phi}}-\boldsymbol{\phi})) P_{n}^{m}(\cos \overline{\boldsymbol{\theta}}) P_{n}^{m}(\cos \boldsymbol{\theta}) \frac{\boldsymbol{\rho}^{n}}{R_{s}^{n+1}}, R_{s} \geq \boldsymbol{\rho}, \\
\frac{-1}{4 \pi \rho} \\
-\sum_{n=1}^{\infty} \sum_{m=0}^{n} \frac{\boldsymbol{\varepsilon}_{m}}{4 \pi} \frac{(n-m) !}{(n+m) !} \cos (m(\overline{\boldsymbol{\phi}}-\boldsymbol{\phi})) P_{n}^{m}(\cos \overline{\boldsymbol{\theta}}) P_{n}^{m}(\cos \boldsymbol{\theta}) \frac{R_{s}^{n}}{\boldsymbol{\rho}^{n+1}}, R_{s}<\boldsymbol{\rho},
\end{array}\right.
$$

where $x=(\rho, \bar{\theta}, \bar{\phi}), \quad s=\left(R_{s}, \theta, \phi\right), \quad P_{n}^{m}(\bullet) \quad$ is the associated Legendre polynomial, the superscripts of $i$ and $e$ denote the interior and exterior regions, respectively, and $\varepsilon_{m}$ is the Neumann factor which defined by,

$$
\varepsilon_{m}=\left\{\begin{array}{l}
1, m=0, \\
2, m=1,2, \cdots \infty .
\end{array}\right.
$$

As mentioned in [8], the interior and exterior Green's functions can satisfy the homogeneous Dirichlet boundary conditions if the image source is correctly selected. The closed-form Green's functions for both interior and exterior problems are written to be the same form

$$
G\left(x, s ; s^{\prime}\right)=\frac{1}{4 \pi}\left(\frac{-1}{|x-s|}+\frac{R_{s}}{a} \frac{1}{\left|x-s^{\prime}\right|}\right), x \in D,
$$

where $a$ is the radius of the sphere, $s=\left(R_{s}, \pi / 2,0\right)$, and $s^{\prime}$ is the image source and its position is at $\left(a^{2} / R_{s}, \pi / 2,0\right)$ as shown in Fig. 2 . It is interesting that the formulae for the location of image are the same as the 2-D case [6]. However, the magnitude of strength $\left(R_{s} / a\right)$ is different from the 2-D case [6].

In order to match the inner and outer homogenous Dirichlet boundary conditions, the image relation between the source point and successive image points yields

$$
\begin{aligned}
& R_{1}=\frac{b^{2}}{R_{s}}, \quad R_{5}=\frac{b^{2}}{R_{s}} \frac{b^{2}}{a^{2}} \ldots \ldots ., R_{4 i-3}=\frac{b^{2}}{R_{s}}\left(\frac{b^{2}}{a^{2}}\right)^{i-1}, \\
& R_{2}=\frac{a^{2}}{R_{s}}, \quad R_{6}=\frac{a^{2}}{R_{s}} \frac{a^{2}}{b^{2}} \ldots \ldots, R_{4 i-2}=\frac{a^{2}}{R_{s}}\left(\frac{a^{2}}{b^{2}}\right)^{i-1}, \\
& R_{3}=\frac{b^{2} R_{s}}{a^{2}}, R_{7}=\frac{b^{2} R_{s}}{a^{2}} \frac{b^{2}}{a^{2}} \ldots, R_{4 i-1}=\frac{b^{2} R_{s}}{a^{2}}\left(\frac{b^{2}}{a^{2}}\right)^{i-1}, \\
& R_{4}=\frac{a^{2} R_{s}}{b^{2}}, R_{8}=\frac{a^{2} R_{s}}{b^{2}} \frac{a^{2}}{b^{2}} \ldots, R_{4 i}=\frac{a^{2} R_{s}}{b^{2}}\left(\frac{a^{2}}{b^{2}}\right)^{i-1} .
\end{aligned}
$$




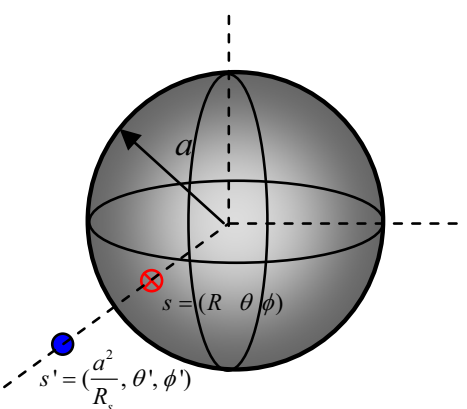

(a)

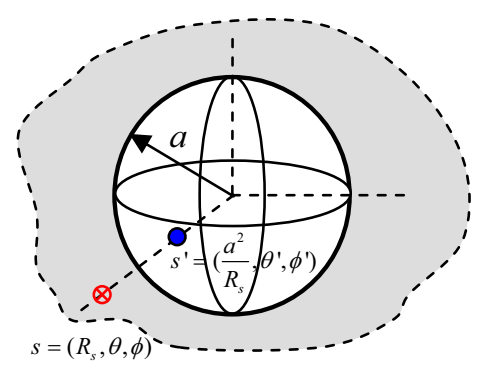

(b)

Figure 2: $\quad$ Sketch of position of image point (a) interior case and (b) exterior case.

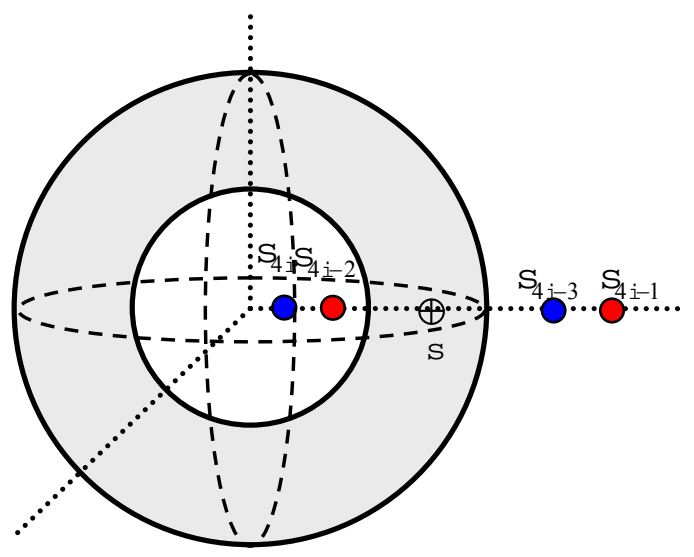

Figure 3: $\quad$ Successive images for an annular problem.

The successive image points for the concentric sphere are shown in Fig. 3. After successive image process, the main part of Green's function is expressed by

$$
G_{m}(x, s)=\frac{1}{4 \pi}\left\{\frac{-1}{|x-s|}+\lim _{N \rightarrow \infty} \sum_{i=1}^{N}\left[\frac{w_{4 i-3}}{\left|x-s_{4 i-3}\right|}+\frac{w_{4 i-2}}{\left|x-s_{4 i-2}\right|}-\frac{w_{4 i-1}}{\left|x-s_{4 i-1}\right|}-\frac{w_{4 i}}{\left|x-s_{4 i}\right|}\right]\right\},
$$

where $w$ is the weighting of image point which is determined by [8]

$$
\begin{aligned}
& w_{1}=\frac{R_{1}}{b}=\frac{b}{R_{s}}, w_{5}=\frac{a R_{5}}{b^{2}}=\frac{b}{R_{s}}\left(\frac{b}{a}\right), \quad w_{9}=\frac{a^{2} R_{9}}{b^{3}}=\frac{b}{R_{s}}\left(\frac{b}{a}\right)^{2} \cdots \cdots, w_{4 i-3}=\frac{b}{R_{s}}\left(\frac{b}{a}\right)^{i-1}, \\
& w_{2}=\frac{a}{R_{s}}, \quad w_{6}=\frac{a^{2}}{b R_{s}}=\frac{a}{R_{s}}\left(\frac{a}{b}\right), \quad w_{10}=\frac{a^{3}}{b^{2} R_{s}}=\frac{a}{R}\left(\frac{a}{b}\right)^{2} \cdots \cdots, w_{4 i-2}=\frac{a}{R_{s}}\left(\frac{a}{b}\right)^{i-1}, \\
& w_{3}=\frac{a R_{3}}{b R_{s}}=\frac{b}{a}, w_{7}=\frac{a^{2} R_{7}}{b^{2} R_{s}}=\frac{b}{a}\left(\frac{b}{a}\right), \quad w_{11}=\frac{a^{3} R_{11}}{b^{3} R_{s}}=\frac{b}{a}\left(\frac{b}{a}\right)^{2} \cdots, \quad w_{4 i-1}=\frac{b}{a}\left(\frac{b}{a}\right)^{i-1} \text {, } \\
& w_{4}=\frac{a}{b}, \quad w_{8}=\frac{a^{2}}{b^{2}}=\frac{a}{b}\left(\frac{a}{b}\right), \quad w_{12}=\frac{a^{3}}{b^{3}}=\frac{a}{b}\left(\frac{a}{b}\right)^{2} \cdots \cdots \cdots, w_{4 i}=\frac{a}{b}\left(\frac{a}{b}\right)^{i-1} .
\end{aligned}
$$




\subsubsection{Satisfaction of the boundary condition by using interpolation functions}

We set $G_{m}(x, s)$ to be the main part of the Green's function in Eq.(9). Unfortunately, $G_{m}(x, s)$ in Eq.(9) can not simultaneously satisfy both the inner and outer boundary conditions of $G_{m}\left(x_{a}, s\right)=G_{m}\left(x_{b}, s\right)=0$, where $x_{a}=(a, \bar{\theta}, \bar{\phi}), x_{b}=(b, \bar{\theta}, \bar{\phi}), 0 \leq \bar{\theta} \leq \pi, 0 \leq \bar{\phi} \leq 2 \pi$. In order to satisfy both the inner and outer boundary conditions, an alternative method is introduced such that we have

$$
G(x, s)=G_{m}(x, s)-\left(\frac{b(\rho-a)}{\rho(b-a)}\right) G_{m}\left(x_{b}, s\right)-\left(\frac{a(b-\rho)}{\rho(b-a)}\right) G_{m}\left(x_{a}, s\right), a \leq \rho \leq b,
$$

where $(b(\rho-a) / \rho(b-a))$ and $(a(b-\rho) / \rho(b-a))$ are the interpolation functions, $G_{m}\left(x_{b}, s\right)=-\left(\frac{a}{b}\right)^{N} \frac{1}{b}$ and $G_{m}\left(x_{a}, s\right)=\frac{-1}{R_{s}}\left(\frac{a}{b}\right)^{N}$. Therefore, Eq.(11) can be simplified to

$$
\begin{aligned}
G(x, s)=\frac{1}{4 \pi} & \left\{\frac{-1}{|x-s|}+\lim _{N \rightarrow \infty} \sum_{i=1}^{N}\left[\frac{w_{4 i-3}}{\left|x-s_{4 i-3}\right|}+\frac{w_{4 i-2}}{\left|x-s_{4 i-2}\right|}-\frac{w_{4 i-1}}{\left|x-s_{4 i-1}\right|}-\frac{w_{4 i}}{\left|x-s_{4 i}\right|}\right]\right. \\
& \left.+C+D \frac{1}{\rho}\right\}
\end{aligned}
$$

where $C=\left(\frac{a}{b}\right)^{N}\left[\frac{a-R_{s}}{R_{s}(a-b)}\right]$ and $D=\left(\frac{a}{b}\right)^{N}\left[\frac{a\left(R_{s}-b\right)}{R_{s}(a-b)}\right]$. Equation (12) indicates that not only image singularities at $s_{4 i-3}, s_{4 i-2}, s_{4 i-1}$ and $s_{4 i}$, but also one singularity at the origin and one rigid body term for one singularity at infinity are required. The Green's function in Eq.(12) satisfies the governing equation and boundary conditions at the same time. It is found that a conventional MFS always loses a free constant and completeness may be questionable.

\subsubsection{Satisfaction of boundary conditions to determine two singularity strengths at the origin and infinity}

After successive image process, the final two image locations freeze at the origin and infinity. There are two strengths of singularities to be determined. Therefore, the Green's function is rewritten as

$$
\begin{aligned}
G(x, s)= & \frac{1}{4 \pi}\left\{\frac{-1}{|x-s|}+\lim _{N \rightarrow \infty} \sum_{i=1}^{N}\left[\frac{w_{4 i-3}}{\left|x-s_{4 i-3}\right|}+\frac{w_{4 i-2}}{\left|x-s_{4 i-2}\right|}-\frac{w_{4 i-1}}{\left|x-s_{4 i-1}\right|}-\frac{w_{4 i}}{\left|x-s_{4 i}\right|}\right]\right. \\
& \left.+c(N)+\frac{d(N)}{\rho}\right\},
\end{aligned}
$$

where the final two images locate at the center of sphere and infinity with strength of $c(N)$ and $d(N)$, respectively, which can be analytically and numerically determined by matching the inner and outer boundary conditions.

After matching the inner and outer boundary conditions, the unknown coefficients $c(N)$ and $d(N)$ are determined by using the numerical method and analytical approach are shown in Fig 4. Agreement is made. 

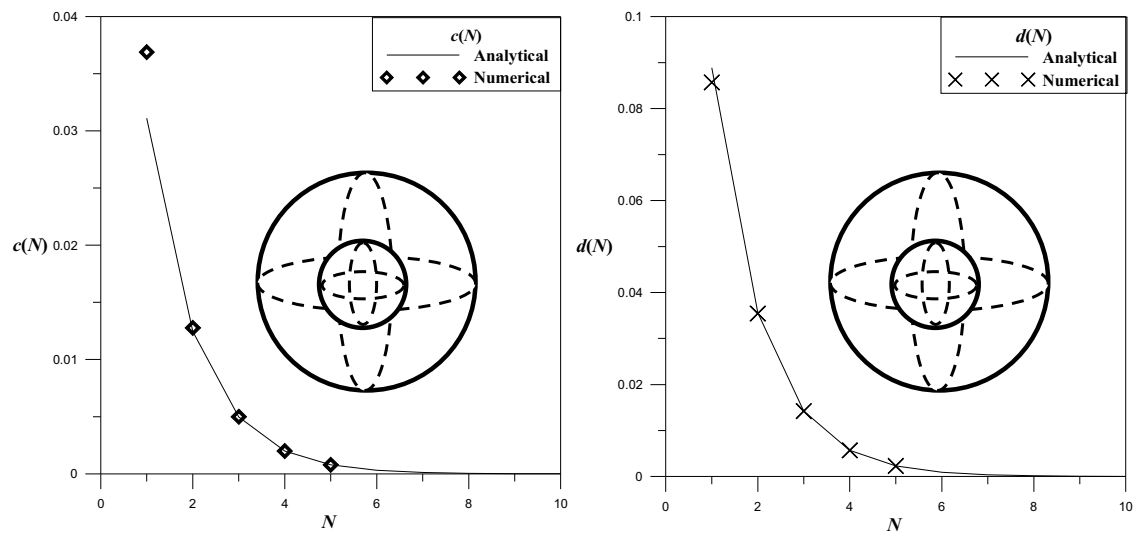

Figure 4: $\quad$ Coefficients of $c(N)$ and $d(N)$ versus $N$ for the fixed-fixed case.

\subsection{The conventional MFS [2]}

In the method of fundamental solutions, the Green's function $G(x, s)$ is superimposed by using the fundamental solutions $U(x, s)$, as follows:

$$
G(x, s)=\frac{-1}{4 \pi r}+\sum_{j=1}^{N_{M}} \alpha_{j} U\left(x, s_{j}\right), x \in D,
$$

where the $N_{M}$ is the number of source points which are distributed outside the domain, $\alpha_{j}$ is the $j^{\text {th }}$ unknown coefficient. By similarly matching the boundary conditions in Eq.(2), the unknown coefficient $\alpha_{j}$ can be determined. Then, we have a numerical solution. By comparing Eq.(13) with Eq.(14), the image method of Eq.(13) can be seen as a special MFS of Eq.(14) with optimal locations and specified strengths for the singularities except the two strengths at two frozen points.

\section{Derivation of the Green's function for the domain bounded by concentric spheres by using the Trefftz method}

The problem of a concentric sphere in Fig. 5 can be decomposed into two parts. One is an infinite space with a concentrated source (fundamental solution) in Fig. 5(a) and the other is subject to specified boundary conditions as shown in Fig. 5(b). The first-part solution can be obtained from the fundamental solution. Here, the second part is solved by using the Trefftz method. The solution can be superposed by using the Trefftz bases as shown below:

$$
G_{T}(x, s)=\sum_{j=1}^{N_{T}} p_{j} \Phi_{j}
$$




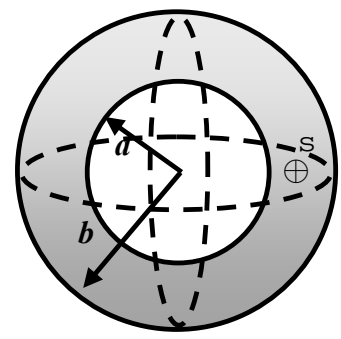

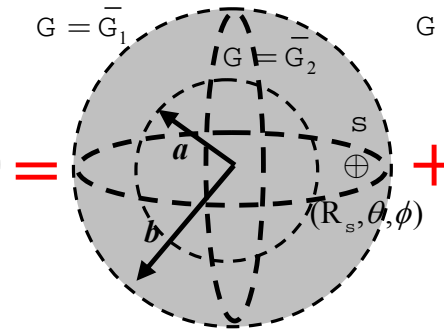

(a)

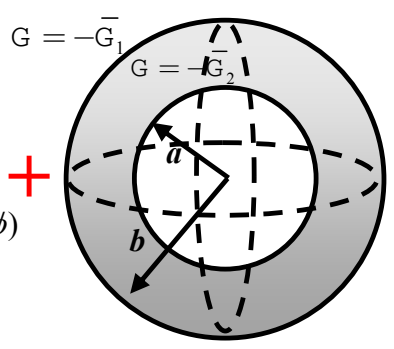

(b)

Figure 5: Sketch of the superposition approach. (a) An infinite space with a concentrated source. (b) A concentric sphere subject to the Dirichlet boundary condition.

where $p_{j}$ is the weighting, $\Phi_{j}$ is the $j^{\text {th }}$ T-complete function and $N_{T}$ is the number of T-complete functions.

Here, the T-complete functions are given as $1, \rho^{n} P_{n}^{m}(\cos \bar{\theta}) \cos (m \bar{\phi})$ and $\rho^{n} P_{n}^{m}(\cos \bar{\theta}) \sin (m \bar{\phi})$ for the interior case and $1 / \rho, \rho^{-(n+1)} P_{n}^{m}(\cos \bar{\theta}) \cos (m \bar{\phi})$ and $\rho^{-(n+1)} P_{n}^{m}(\cos \bar{\theta}) \sin (m \bar{\phi})$ for the exterior case. The second-part solution can be represented by

$$
\begin{aligned}
G_{T}(x, s)= & A_{00}+\frac{B_{00}}{\rho}+\sum_{n=1}^{\infty} \sum_{m=0}^{n}\left[A_{n m} \rho^{n} P_{n}^{m}(\cos \bar{\theta}) \cos (m \bar{\phi})+B_{n m} \rho^{-(n+1)} P_{n}^{m}(\cos \bar{\theta}) \cos (m \bar{\phi})\right. \\
& \left.+C_{n m} \rho^{n} P_{n}^{m}(\cos \bar{\theta}) \sin (m \bar{\phi})+D_{n m} \rho^{-(n+1)} P_{n}^{m}(\cos \bar{\theta}) \sin (m \bar{\phi})\right],
\end{aligned}
$$

where $A_{00}, B_{00}, A_{n m}, B_{n m}, C_{n m}$ and $D_{n m}$ are unknown coefficients. By matching the boundary conditions, the unknown coefficients can be determined. Then, the series-form Green's function is obtained by superimposing the solutions of $U(x, s)$ and $G_{T}(x, s)$ as shown below:

$$
\begin{aligned}
G(x, s)= & \frac{-1}{4 \pi|x-s|}+A_{00}+\frac{B_{00}}{\rho} \\
& +\sum_{n=1}^{\infty} \sum_{m=0}^{n}\left[A_{n m} \rho^{n} P_{n}^{m}(\cos \bar{\theta}) \cos (m \bar{\phi})+B_{n m} \rho^{-(n+1)} P_{n}^{m}(\cos \bar{\theta}) \cos (m \bar{\phi})\right. \\
& \left.+C_{n m} \rho^{n} P_{n}^{m}(\cos \bar{\theta}) \sin (m \bar{\phi})+D_{n m} \rho^{-(n+1)} P_{n}^{m}(\cos \bar{\theta}) \sin (m \bar{\phi})\right],
\end{aligned}
$$

where the unknown coefficients are obtained as shown below:

$$
\left\{\begin{array}{l}
A_{00} \\
B_{00}
\end{array}\right\}=\left\{\begin{array}{c}
\frac{R_{s}-a}{4 \pi R_{s}(b-a)} \\
\frac{a\left(b-R_{\xi}\right)}{4 \pi R_{s}(b-a)}
\end{array}\right\},
$$




$$
\begin{aligned}
& \left\{\begin{array}{c}
A_{n m} \\
B_{n m}
\end{array}\right\}=\left\{\begin{array}{l}
\frac{\varepsilon_{m}}{4 \pi} \frac{(n-m) !}{(n+m) !}\left[\frac{R_{s}^{2 n+1}-a^{2 n+1}}{R_{s}^{n+1}\left(b^{2 n+1}-a^{2 n+1}\right)}\right] P_{n}^{m}(\cos \theta) \cos (m \phi) \\
\frac{\varepsilon_{m}}{4 \pi} \frac{(n-m) !}{(n+m) !}\left[\frac{a^{2 n+1}\left(b^{2 n+1}-R_{s}^{2 n+1}\right)}{R_{s}^{n+1}\left(b^{2 n+1}-a^{2 n+1}\right)}\right] P_{n}^{m}(\cos \theta) \cos (m \phi)
\end{array}\right\}, \\
& \left\{\begin{array}{l}
C_{n m} \\
D_{n m}
\end{array}\right\}=\left\{\begin{array}{l}
\frac{\varepsilon_{m}}{4 \pi} \frac{(n-m) !}{(n+m) !}\left[\frac{R_{s}^{2 n+1}-a^{2 n+1}}{R_{s}^{n+1}\left(b^{2 n+1}-a^{2 n+1}\right)}\right] P_{n}^{m}(\cos \theta) \sin (m \phi) \\
\frac{\varepsilon_{m}}{4 \pi} \frac{(n-m) !}{(n+m) !}\left[\frac{a^{2 n+1}\left(b^{2 n+1}-R_{s}^{2 n+1}\right)}{R_{s}^{n+1}\left(b^{2 n+1}-a^{2 n+1}\right)}\right] P_{n}^{m}(\cos \theta) \sin (m \phi)
\end{array}\right\},
\end{aligned}
$$

It is interesting to find that Eq.(18) can be included into Eq.(19) since we introduce the Neumann factor and set $n=m=0$. We will prove the equivalence of solutions derived by using the Trefftz method and the image method (special MFS) in the next section.

\section{Mathematical equivalence for the solutions derived by the MFS and Trefftz method}

\subsection{Method of fundamental solutions (image method)}

The image method can be seen as a special case of the MFS, since its singularities are located outside the domain. The Green's function of Eq.(13) can be expanded into a series form by separating the field point $x$ and source point $s$ for the fundamental solution in the spherical coordinates of Eq.(5) as shown below:

$$
\begin{aligned}
G(x, s) & =\frac{1}{4 \pi}\left[\frac{-1}{|x-s|}+\frac{R_{s}-a}{R_{s}(b-a)}+\frac{a\left(b-R_{s}\right)}{R_{s} \rho(b-a)}\right] \\
& +\sum_{i=1}^{\infty} \sum_{n=1}^{\infty} \sum_{m=0}^{n} \frac{\varepsilon_{m}}{4 \pi} \frac{(n-m) !}{(n+m) !}\left[w_{4 i-3}\left(\frac{\rho^{n}}{R_{4 i-3}^{n+1}}\right)+w_{4 i-2}\left(\frac{R_{4 i-2}^{n}}{\rho^{n+1}}\right)\right. \\
& \left.-w_{4 i-1}\left(\frac{\rho^{n}}{R_{4 i-1}^{n+1}}\right)-w_{4 i}\left(\frac{R_{4 i}^{n}}{\rho^{n+1}}\right)\right] \cos [m(\bar{\phi}-\phi)] P_{n}^{m}(\cos \bar{\theta}) P_{n}^{m}(\cos \theta) .
\end{aligned}
$$

By using Eqs. (8) and (10), the series containing four geometry series with the same ratio of $(a / b)^{2 n+1}$ which is smaller than one in Eq.(13) can be rearranged into

$$
\begin{aligned}
G(x, s)= & \frac{1}{4 \pi}\left[\frac{-1}{|x-s|}+\frac{R_{s}-a}{R_{s}(b-a)}+\frac{a\left(b-R_{s}\right)}{R_{s} \rho(b-a)}\right] \\
+ & \sum_{n=1}^{\infty} \sum_{m=0}^{n} \frac{\varepsilon_{m}}{4 \pi} \frac{(n-m) !}{(n+m) !}\left[\frac{R_{s}^{2 n+1} \rho^{2 n+1}-a^{2 n+1} \rho^{2 n+1}+a^{2 n+1} b^{2 n+1}-a^{2 n+1} R_{s}^{2 n+1}}{R_{s}^{n+1} \rho^{n+1}\left(b^{2 n+1}-a^{2 n+1}\right)}\right] \\
& \cos [m(\bar{\phi}-\phi)] P_{n}^{m}(\cos \bar{\theta}) P_{n}^{m}(\cos \theta),
\end{aligned}
$$


after expanding all the image singularities of $-1 / r$ functions. It is interesting to find that the optimal location may not be the expansion type of Fig. 6(a) or angular distribution of Fig. 6(b) but a lump singularity in one radial direction shown in Fig. 6(c) as mentioned by Antunes [9]. In this paper, our image location in the MFS only distribute along the radial direction which agrees with the optimal location in [9]. This finding agrees with the experience in the annular case [6] and the present case of a concentric sphere.

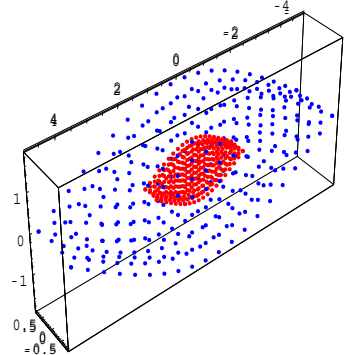

(a) Expansion

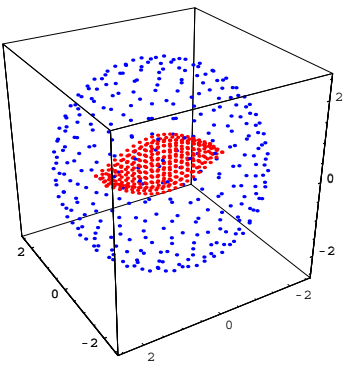

(b) Sphere

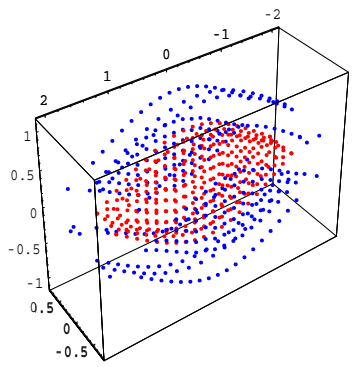

(c) Lump (Optimal case)

Figure 6: Optimal locations of source distribution for the MFS [9].

\subsection{Trefftz method}

Substitution of Eqs.(18)-(20) into Eq.(17) yields

$$
\begin{aligned}
G(x, s)= & \frac{1}{4 \pi}\left[\frac{-1}{|x-s|}+\frac{R_{s}-a}{R_{s}(b-a)}+\frac{a\left(b-R_{s}\right)}{R_{s} \rho(b-a)}\right] \\
+ & \sum_{n=1}^{\infty} \sum_{m=0}^{n} \frac{\varepsilon_{m}}{4 \pi} \frac{(n-m) !}{(n+m) !}\left[\frac{R_{s}^{2 n+1} \rho^{2 n+1}-a^{2 n+1} \rho^{2 n+1}+a^{2 n+1} b^{2 n+1}-a^{2 n+1} R_{s}^{2 n+1}}{R_{s}^{n+1} \rho^{n+1}\left(b^{2 n+1}-a^{2 n+1}\right)}\right] \\
& \cos [m(\bar{\phi}-\phi)] P_{n}^{m}(\cos \bar{\theta}) P_{n}^{m}(\cos \theta) .
\end{aligned}
$$

After comparing Eq.(22) with Eq.(23), it is found that the two solutions, Eqs. (13) and (17) are proved to be mathematically equivalent by using the addition theorem when the number of images and the number of Trefftz bases are both infinite. To verify the Trefftz and image solutions, the conventional MFS is utilized for comparison. The distribution of collocation nodes and images (sources of MFS) is shown in Fig. 7. Contour plots by using the three approaches are shown in Fig. 8. Good agreement is observed.

\section{Concluding remarks}

In this paper, not only the image method (a special MFS) but also the Trefftz method was employed to solve the Green's function of 3-D Laplace problems bounded by concentric sphere. The two solutions using the Trefftz method and MFS for the case were proved to be mathematically equivalent by using the 


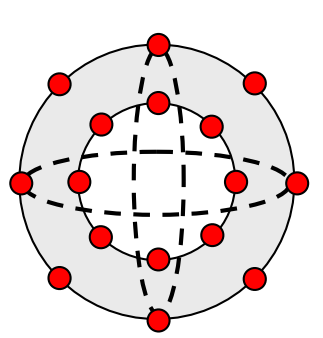

Source point

(a)

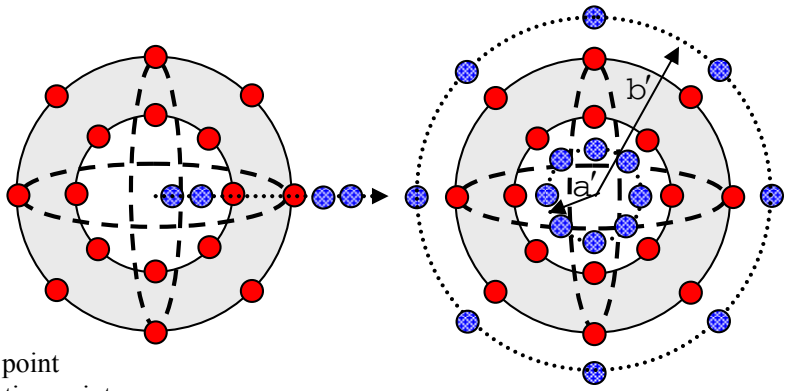

(b)

(c)

Figure 7: $\quad$ Sketches of (a) Trefftz method, (b) image method (special MFS, radial distribution of singularities) and (c) conventional MFS (angular distribution of singularities).

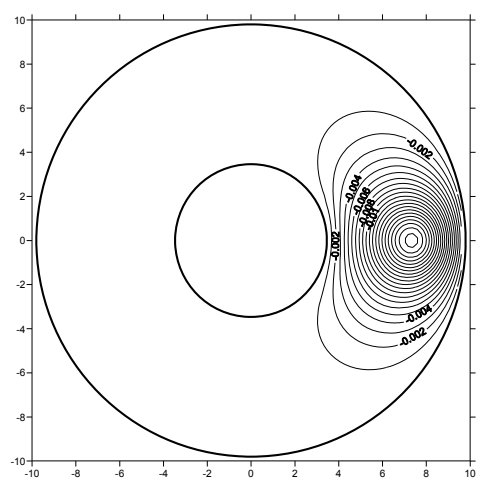

(a) Trefftz method

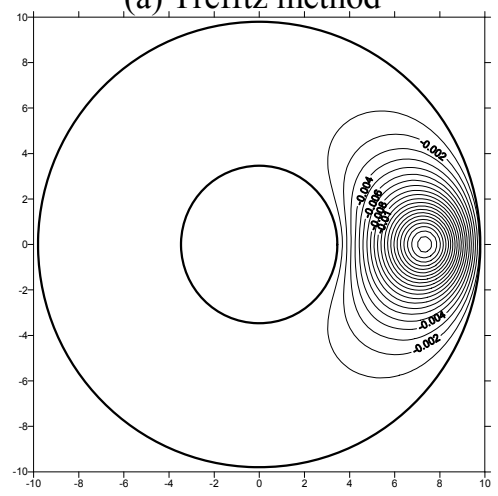

(c) MFS

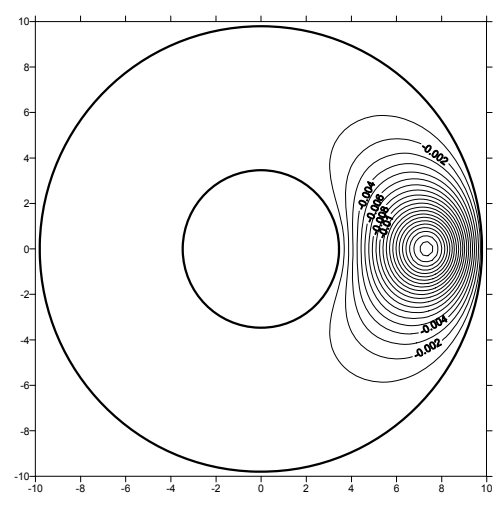

(b) Image method

Figure 8: Contour plots by using three approaches, (a) Trefftz method, (b) image method and (c) MFS ( $z=2$ plane). 
addition theorem or so-called degenerate kernel. Also, the solution of image method shows the existence of the free constant which is always overlooked in the conventional MFS although it becomes zero as the number of images approaches infinity. Finally, we also found the final two frozen image points at the origin and infinity where their strengths can be determined numerically and analytically in a consistent manner. Contour plots by using the three approaches, Trefftz method, image method and conventional MFS, agree well.

\section{References}

[1] Kita E. Kamiya N. Trefftz method: an overview. Adv Engng Softw 1995;24:3-12.

[2] Kupradze VD. A method for the approximate solution of limiting problems in mathematical physics. Computat Math Math Phys 1964;4:199-205.

[3] Bogomolny A. Fundamental solutions method for elliptic boundary value problems. SIAM J Numer Anal 1985;22(4):644-669.

[4] Li ZC. Lu TT. Hu HY. Cheng AHD. Trefftz and collocation methods. Boston-Southampton: WIT Press; 2007.

[5] Chen JT. Wu CS. Lee YT. Chen KH. On the equivalence of the Trefftz method and method of fundamental solutions for Laplace and biharmonic equations. Computers and Mathematics with Applications 2007;53:851-879.

[6] Chen JT. Lee YT. Yu SR. Shieh SC. 2009, Equivalence between Trefftz method and method of fundamental solution for the annular Green's function using the addition theorem and image concept, Engineering Analysis with Boundary Elements, Vol.33, pp.678-688.

[7] Schaback R. Adaptive numerical solution of MFS systems. A plenary talk at the first Inter Workshop on the Method of Fundamental Solution, Ayia Napa, Cyprus, June 11-13; 2007.

[8] Chen JT. Wu CS. Alternative derivations for the Poisson integral formula. Int. J Math Educ. Sci. Technol 2006;37:165-185.

[9] Antunes PRS. The Method of Fundamental Solutions applied to the eigenproblems in partial differential equations, Ph.D. Dissertation, IST, Portugal, 2008. 